\title{
On the stability of high- $K$ isomers in the second well of actinide nuclei
}

\author{
H.L. Liu ${ }^{1,2}$, F.R. Xu ${ }^{1,3}$, Y. Sun ${ }^{2,3}$, P.M. Walker ${ }^{4}$, and R. Wyss ${ }^{5}$ \\ 1 School of Physics, and State Key Laboratory of Nuclear Physics and Technology, Peking University, Beijing 100871, China \\ 2 Department of Physics, Shanghai Jiao Tong University, Shanghai 200240, China \\ 3 Center for Theoretical Nuclear Physics, National Laboratory for Heavy Ion Physics, Lanzhou 730000, China \\ 4 Department of Physics, University of Surrey, Guildford GU2 7XH, UK \\ 5 AlbaNova University Center, KTH (Royal Institute of Technology), S-106 91 Stockholm, Sweden
}

Received: date / Revised version: date

\begin{abstract}
Actinide nuclei are found to be good candidates for the formation of high angular momentum, broken-pair excitations in the second minimum of the potential-energy surface. Configuration-constrained calculations of the energy surfaces, including reflection asymmetry, give predictions of the properties of high- $K$ states in the second well. In addition to excitation energies, spins and parities, the calculations indicate increased barriers towards fission, consistent with the extended half-lives observed experimentally.
\end{abstract}

PACS. 2 1.10.-k, 21.60.-n, 23.20.Lv

Broken-pair excitations can lead to metastable states with an excitation of the quantum degree of freedom, $K$, that is the angular-momentum projection onto the symmetry axis of a deformed nucleus. A high- $K$ excitation can result in highly forbidden decays to low- $K$ states, due to the $K$ selection rule [1], leading to the formation of longlived high- $K$ isomers, which exist widely in the nuclear chart [2]. From the excitation energies and configurations of the isomers, insight can be gained into the shell structure of nucleon orbits, since quasiparticle excitations are closely related to the orbital fillings of the unpaired nucleons and their intrinsic angular momenta.

In atomic nuclei there is another kind of metastable state that owes its existence to a second well of the potentialenergy surface (PES) of the nucleus with a highly elongated shape (where the first well generates the ground state). In the $Z>90$ actinide region, the shape-trapping state decays mostly by spontaneous fission (SF), and hence is usually called a 'fission isomer'. The emergence of the second energy minimum is due to the special shell structure of nucleon orbits where clear shell gaps exist at large deformation. Because of very different shapes and a significant barrier between the first and second minima, the decay from the fission isomer to the ground state is inhibited. Indeed, actinide nuclei are well known for fission isomers $[3,4]$ with highly elongated ('superdeformed') shapes, having major-to-minor axis ratios of about $2: 1$.

It is natural to consider the possibility of high- $K$ isomerism in the second well. The superdeformed axiallysymmetric shape is an excellent condition for the conser-

Send offprint requests to: vation of the $K$ quantum number. In addition, a high- $K$ configuration at the fission shape could increase the stability of an isomer against fission, in a similar manner to the fission inhibition in the first well [5]. This is in accord with experimental observations of longer-lived fission isomers, excited within the second well [3]. We call the state associated with the double isomerism of high $K$ and shape a 'high- $K$ fission isomer'.

As early as the 1970s, Limkilde and Sletten explained the observed $T_{1 / 2}=5$ ns state in ${ }^{238} \mathrm{Pu}$ as a $K$ isomer [6], contrasting with the $0.5 \mathrm{~ns} 0^{+}$fission isomer. The experimental energy relative to the $0^{+}$fission isomer was consistent with twice the pairing gap, as expected for a broken-pair excitation. Recently, high- $K$ fission isomers were investigated theoretically using blocking calculations with fixed second-well deformations, using the Gogny force [7]. Up to now, only in a few even-even actinide nuclei, ${ }^{238} \mathrm{U},{ }^{236,238,242} \mathrm{Pu}$ and ${ }^{240,242,244} \mathrm{Cm}$, has experimental evidence for high- $K$ fission isomers been obtained, and even then only half-lives and excitation energies have been measured [4]. Their spins and parities have not been determined due to the lack of observation of $\gamma$-ray transitions to known states, which could well be $K$ forbidden. Therefore, additional experimental measurements and further theoretical investigations are still needed. Configuration-constrained PES calculations that consider shape polarization effects from quasiparticle excitations can give detailed insight into the structure of these isomers.

The configuration-constrained PES model [8] has been widely used to calculate high- $K$ isomeric states in various mass regions including the superheavy [5] and drip- 
line [9] regions. In these extreme regions of nuclei, where ground-state half-lives become short, the ability of excited, broken-pair states to provide extra stability is remarkable, at least in the sense that the isomers can have significantly longer half-lives than their respective ground states.

In the previous works, PESs were calculated in the $\left(\beta_{2}, \gamma, \beta_{4}\right)$ deformation space. These three deformation degrees of freedom are usually sufficient for normally deformed nuclei. However, it has been found that other highmultipole deformations, especially those with reflection asymmetry, are important for understanding the large deformations of heavy nuclei (see, e.g., Refs. [10-12]). In the actinide mass region, for example, fission barriers minimized with respect to reflection asymmetry can be significantly lower than those assuming reflection symmetry [13], while these nuclei seem to be rigid against $\gamma$ distortion [3]. To describe properly the second minima of actinide nuclei, we have modified the configuration-constrained PES calculation to take account of the deformation parameters $\left(\beta_{2}, \beta_{3}, \beta_{4}, \beta_{5}\right)$. Single-particle levels are obtained from the axial reflection-asymmetric Woods-Saxon potential with the set of universal parameters [14]. For the pairing correlation, particle-number projection is approximated by the Lipkin-Nogami method [15], with the pairing strength determined by the average-gap method [16]. The total energy of a nucleus consists of a macroscopic part that is obtained with the standard liquid-drop model [17] and a microscopic part which is calculated by the Strutinsky approach [18].

In the configuration-constrained PES calculation, the unpaired nucleon orbits which specify a given configuration [8] are blocked in each point of the selected deformation lattice. This has been achieved by calculating and identifying the average Nilsson quantum numbers for every orbit involved in the configuration. The process is the same as that in the $\left(\beta_{2}, \gamma, \beta_{4}\right)$ space [8] except that, for reflection-asymmetric deformation, parity is no longer conserved, while $\Omega$, the nucleon spin projection onto the symmetry axis, is a good quantum number. We limit our PES calculation to the deformation region of $\left|\beta_{3}\right| \leq 0.3$ because higher $\beta_{3}$ deformation can cause strong mixing of orbits, leading to difficulty in tracing the given orbits. This is similar to the calculation for nonaxial deformations, where tracing orbits at large $\gamma$ deformation needs special attention [8]. Nevertheless, in the present case, where large $\beta_{3}$ deformations are involved in fission, the restriction to $\beta_{3} \leq 0.3$ is a critical aspect which will have to be addressed in future work if quantitative fission probabilities are to be obtained. The configuration-constrained calculation gives the PES for a given configuration in the $\left(\beta_{2}, \beta_{3}\right)$ plane. At each $\left(\beta_{2}, \beta_{3}\right)$ grid point, the energy has been minimized with respect to $\beta_{4}$ and $\beta_{5}$ deformations. Such PESs can show the shape changes due to the polarization of unpaired nucleons.

The $0^{+}$fission isomers are calculated first. Fig. 1 shows comparisons between calculated and measured properties of the $0^{+}$fission isomers in $\mathrm{U}, \mathrm{Pu}$ and $\mathrm{Cm}$ isotopes $(Z=$ 92,94 and 96 , respectively). The overall agreement is reasonably good. Note that the experimental energies were es- timated from excitation function measurements [3] except ${ }^{236,238} \mathrm{U}$ for which $\gamma$ transitions were observed from the $0^{+}$ fission isomers to states in the first well [20,21], allowing accurate determination of excitation energies. Moreover, for ${ }^{238} \mathrm{U}$, the $E 0$ conversion-electron transition was observed between the $0^{+}$fission isomer and ground state [21]. One can see in Fig. 1 that our calculations reproduce well the excitation energies in ${ }^{236,238} \mathrm{U}$. The present calculations can also reproduce the deduced $\beta_{2}$ deformations (see Fig. 1) which are almost constant for each isotopic chain with $N=140-152: \beta_{2} \approx 0.23$ for the first minimum and $\beta_{2} \approx 0.65$ for the second minimum.

In Table 1, we present the calculations for the lowestlying two-quasiparticle states within the second well, including comparison with available experimental data [4]. Only the high- $K\left(\Omega_{1}+\Omega_{2}\right)$ couplings are illustrated, since these account for the states that may form isomers, with $K$-inhibited decays [2]. We see that there are several different high- $K$ configurations in each case. Energies relative to the second minima are as low as about $1 \mathrm{MeV}$ for the twoquasineutron states. This is in accord with the calculations of Ref. [7]. The calculated excitation energies (relative to corresponding ground states) agree with experimental data to within $\approx 400 \mathrm{keV}$ for the observed high- $K$ fission isomers. It should be mentioned that in most cases experimental energies are approximate, obtained from excitation function measurements [3]. In principle, the lowest-energy high- $K$ state is the most likely one to be an isomer due to the $K$ inhibition between the high- $K$ state and the rotational states built upon the $K=0$ fission isomer. However, there exist several low-lying high- $K$ configurations with calculated energies close to each other for each nucleus. With energy uncertainties $\approx 200 \mathrm{keV}$, the calculations cannot reliably determine which is the lowest-energy high- $K$ state that would be responsible for the observed isomer. Experimentally, the spins and parities of the observed high- $K$ fission isomers are unknown. Therefore, any of the low-lying high- $K$ configurations would be possible for an observed $K$ and shape 'doubly isomeric' state in the nucleus. Further experimental information is necessary for the determination of the configurations of the observed high- $K$ fission isomers. Their excitation energies and configurations can provide detailed knowledge of the nuclear single-particle potential and shell gaps for heavy nuclei in the second well.

Our calculated deformations for the high- $K$ states are similar to those of the $0^{+}$fission isomers, justifying the calculations with fixed deformation in Ref. [7]. The rigid, superdeformed, axially-symmetric shapes provide good conditions for the formation of high- $K$ isomers. Usually, a large axial deformation is less susceptible to shape fluctuation towards triaxiality, that would induce $K$ mixing and hence reduce the $K$ inhibition of the decay. Furthermore, the combined action of the shape and $K$ isomerism severely inhibits $\gamma$ decay into states in the first well, thus explaining, at least in part, the absence of such observations.

Besides excitation energy, another observable known for the isomers is half-life, listed in Table 1 . The $0^{+}$fis- 
Table 1. Calculated deformations and excitation energies (relative to corresponding ground states) for several low-lying brokenpair states within the second well, compared with available experimental data [4]. For all the studied states, the calculations give $\beta_{3} \approx 0$ and $\beta_{5} \approx 0$.

\begin{tabular}{|c|c|c|c|c|c|c|c|c|}
\hline Nuclei & $K^{\pi}$ & Configurations & $\overline{\beta_{2}}$ & $\overline{\beta_{4}}$ & $\begin{array}{l}E_{x}^{\text {cal. }}(\mathrm{keV}) \\
\end{array}$ & $\overline{E_{x}^{\text {expt. }}(\mathrm{keV})}$ & $T_{1 / 2}^{\text {expt. }}$ & Decay Mode \\
\hline \multirow[t]{8}{*}{${ }^{238} \mathrm{U}$} & $0^{+}$ & $0^{+}$Fission Isomer & 0.62 & 0.029 & 2383 & 2558 & $298 \mathrm{~ns}$ & $\begin{array}{l}\% \mathrm{IT} \approx 95 \\
\% \mathrm{SF} \approx 5\end{array}$ \\
\hline & $7^{-}$ & $\nu 9 / 2[734] \otimes \nu 5 / 2[622]$ & 0.61 & 0.022 & 3258 & $<3558$ & $>1 \mathrm{~ns}$ & $\mathrm{SF}$ \\
\hline & $7^{-}$ & $\nu 5 / 2[862] \otimes \nu 9 / 2[734]$ & 0.63 & 0.037 & 3344 & & & \\
\hline & $10^{-}$ & $\nu 11 / 2[615] \otimes \nu 9 / 2[734]$ & 0.62 & 0.036 & 3486 & & & \\
\hline & $8^{+}$ & $\nu 11 / 2[615] \otimes \nu 5 / 2[862]$ & 0.62 & 0.043 & 3563 & & & \\
\hline & $6^{-}$ & $\pi 5 / 2[523] \otimes \pi 7 / 2[633]$ & 0.62 & 0.033 & 3899 & & & \\
\hline & $8^{-}$ & $\pi 9 / 2[514] \otimes \pi 7 / 2[633]$ & 0.62 & 0.037 & 3907 & & & \\
\hline & $7^{+}$ & $\pi 9 / 2[514] \otimes \pi 5 / 2[523]$ & 0.63 & 0.042 & 4032 & & & \\
\hline \multirow[t]{5}{*}{${ }^{236} \mathrm{Pu}$} & $0^{+}$ & $0^{+}$Fission Isomer & 0.63 & 0.045 & 2252 & $\approx 3000$ & $37 \mathrm{ps}$ & SF \\
\hline & $8^{+}$ & $\nu 11 / 2[615] \otimes \nu 5 / 2[862]$ & 0.63 & 0.053 & 3671 & $\approx 4000$ & $34 \mathrm{~ns}$ & SF \\
\hline & $8^{-}$ & $\pi 9 / 2[514] \otimes \pi 7 / 2[633]$ & 0.64 & 0.056 & 3833 & & & \\
\hline & $7^{+}$ & $\pi 9 / 2[514] \otimes \pi 5 / 2[523]$ & 0.64 & 0.060 & 3951 & & & \\
\hline & $6^{-}$ & $\pi 5 / 2[523] \otimes \pi 7 / 2[633]$ & 0.64 & 0.055 & 3958 & & & \\
\hline \multirow[t]{7}{*}{${ }^{238} \mathrm{Pu}$} & $0^{+}$ & $0^{+}$Fission Isomer & 0.63 & 0.038 & 2249 & $\approx 2400$ & $0.5 \mathrm{~ns}$ & $\mathrm{SF}$ \\
\hline & $8^{+}$ & $\nu 11 / 2[615] \otimes \nu 5 / 2[862]$ & 0.62 & 0.048 & 3120 & $\approx 3500$ & $5.0 \mathrm{~ns}$ & SF \\
\hline & $7^{-}$ & $\nu 5 / 2[862] \otimes \nu 9 / 2[734]$ & 0.64 & 0.042 & 3299 & & & \\
\hline & $10^{-}$ & $\nu 11 / 2[615] \otimes \nu 9 / 2[734]$ & 0.62 & 0.034 & 3363 & & & \\
\hline & $8^{-}$ & $\pi 9 / 2[514] \otimes \pi 7 / 2[633]$ & 0.64 & 0.048 & 3868 & & & \\
\hline & $6^{-}$ & $\pi 5 / 2[523] \otimes \pi 7 / 2[633]$ & 0.64 & 0.045 & 3982 & & & \\
\hline & $7^{+}$ & $\pi 9 / 2[514] \otimes \pi 5 / 2[523]$ & 0.64 & 0.051 & 4010 & & & \\
\hline \multirow[t]{9}{*}{${ }^{242} \mathrm{Pu}$} & $0^{+}$ & $0^{+}$Fission Isomer & 0.63 & 0.016 & 2341 & $\approx 2200$ & $3.5 \mathrm{~ns}$ & SF \\
\hline & $8^{-}$ & $\nu 9 / 2[734] \otimes \nu 7 / 2[624]$ & 0.62 & 0.013 & 3473 & $<3200$ & $28 \mathrm{~ns}$ & SF \\
\hline & $7^{-}$ & $\nu 9 / 2[734] \otimes \nu 5 / 2[622]$ & 0.63 & 0.026 & 3484 & & & \\
\hline & $6^{+}$ & $\nu 5 / 2[622] \otimes \nu 7 / 2[624]$ & 0.62 & 0.013 & 3587 & & & \\
\hline & $7^{-}$ & $\nu 5 / 2[862] \otimes \nu 9 / 2[734]$ & 0.61 & 0.025 & 3595 & & & \\
\hline & $10^{-}$ & $\nu 11 / 2[615] \otimes \nu 9 / 2[734]$ & 0.65 & 0.037 & 3966 & & & \\
\hline & $8^{-}$ & $\pi 9 / 2[514] \otimes \pi 7 / 2[633]$ & 0.64 & 0.033 & 4120 & & & \\
\hline & $6^{-}$ & $\pi 5 / 2[523] \otimes \pi 7 / 2[633]$ & 0.64 & 0.027 & 4188 & & & \\
\hline & $7^{+}$ & $\pi 9 / 2[514] \otimes \pi 5 / 2[523]$ & 0.65 & 0.036 & 4288 & & & \\
\hline \multirow[t]{4}{*}{${ }^{240} \mathrm{Cm}$} & $0^{+}$ & $0^{+}$Fission Isomer & 0.64 & 0.036 & 1729 & $\approx 2000$ & $10 \mathrm{ps}$ & SF \\
\hline & $8^{+}$ & $\nu 11 / 2[615] \otimes \nu 5 / 2[862]$ & 0.64 & 0.046 & 2602 & $\approx 3000$ & $55 \mathrm{~ns}$ & $\mathrm{SF}$ \\
\hline & $7^{-}$ & $\nu 5 / 2[862] \otimes \nu 9 / 2[734]$ & 0.64 & 0.042 & 2714 & & & \\
\hline & $10^{-}$ & $\nu 11 / 2[615] \otimes \nu 9 / 2[734]$ & 0.64 & 0.032 & 2841 & & & \\
\hline \multirow[t]{5}{*}{${ }^{242} \mathrm{Cm}$} & $0^{+}$ & $0^{+}$Fission Isomer & 0.64 & 0.025 & 1822 & $\approx 1900$ & $40 \mathrm{ps}$ & $\mathrm{SF}$ \\
\hline & $7^{-}$ & $\nu 5 / 2[862] \otimes \nu 9 / 2[734]$ & 0.64 & 0.035 & 2728 & $\approx 2800$ & $180 \mathrm{~ns}$ & $\mathrm{SF}$ \\
\hline & $10^{-}$ & $\nu 11 / 2[615] \otimes \nu 9 / 2[734]$ & 0.65 & 0.030 & 2786 & & & \\
\hline & $8^{+}$ & $\nu 11 / 2[615] \otimes \nu 5 / 2[862]$ & 0.64 & 0.038 & 3034 & & & \\
\hline & $7^{-}$ & $\nu 9 / 2[734] \otimes \nu 5 / 2[622]$ & 0.61 & 0.024 & 3049 & & & \\
\hline \multirow[t]{6}{*}{${ }^{244} \mathrm{Cm}$} & $0^{+}$ & $0^{+}$Fission Isomer & 0.65 & 0.016 & 1947 & $\approx 2200$ & $<5 \mathrm{ps}$ & $\mathrm{SF}$ \\
\hline & $7^{-}$ & $\nu 9 / 2[734] \otimes \nu 5 / 2[622]$ & 0.64 & 0.025 & 3082 & $\approx 3500$ & $>100 \mathrm{~ns}$ & SF \\
\hline & $8^{-}$ & $\nu 9 / 2[734] \otimes \nu 7 / 2[624]$ & 0.64 & 0.013 & 3153 & & & \\
\hline & $6^{+}$ & $\nu 5 / 2[622] \otimes \nu 7 / 2[624]$ & 0.62 & 0.015 & 3364 & & & \\
\hline & $7^{-}$ & $\nu 5 / 2[862] \otimes \nu 9 / 2[734]$ & 0.62 & 0.026 & 3404 & & & \\
\hline & $10^{-}$ & $\nu 11 / 2[615] \otimes \nu 9 / 2[734]$ & 0.66 & 0.034 & 3462 & & & \\
\hline
\end{tabular}

sion isomer in ${ }^{238} \mathrm{U}$ has a significantly longer half-life than those in other actinide nuclei. This is due to a higher fission barrier, as shown in Ref. [3], enabling the observation of competing decays through shape-forbidden $\gamma$ transitions into states within the first well. The $0^{+}$fission isomers in other nuclei decay by spontaneous fission, and all the observed high- $K$ isomers within the second well undergo fission decays. It is seen that the high- $K$ fis- sion isomers have longer half-lives than the corresponding $0^{+}$fission isomers (except possibly for ${ }^{238} \mathrm{U}$, where only a lower-limit half-life is given for the observed high- $K$ fission isomer). In particular, the observed high- $K$ fission isomers in ${ }^{236} \mathrm{Pu}$ and ${ }^{240,242,244} \mathrm{Cm}$ live at least three orders of magnitude longer than the corresponding $0^{+}$fission isomers. The present work seeks a better understanding of this well known but unexpected [3] observation. 


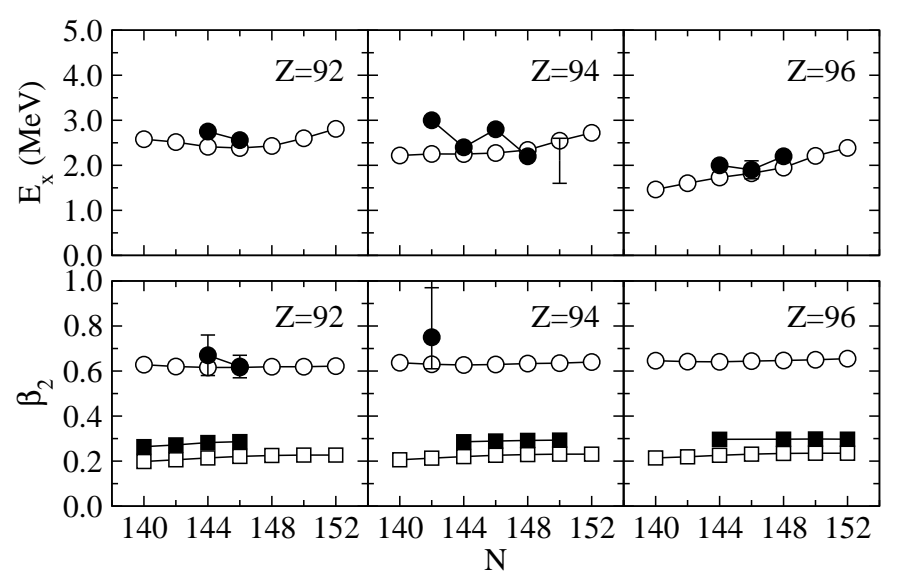

Fig. 1. The upper panel shows the energy differences between the second and first minima. The lower panel displays the $\beta_{2}$ deformations of the first (denoted by squares) and second minima (circles). Open and filled symbols represent calculated and experimental values $[4,19]$, respectively. For ${ }_{94}^{244} \mathrm{Pu}$, the experiment [4] gives an energy range only.

We now consider the calculated second-well PESs. As representatives, states in ${ }^{236} \mathrm{Pu}$ and ${ }^{242} \mathrm{Cm}$ are shown in Fig. 2. The $8^{+}\left(10^{-}\right)$state in ${ }^{236} \mathrm{Pu}\left({ }^{242} \mathrm{Cm}\right)$ has a more rigid (i.e. steeper sided) well than the $8^{-}\left(7^{-}\right)$state. This is because the energies of the two orbits occupied by the unpaired nucleons of the $8^{+}$and $10^{-}$states separate quickly with changing deformation. More importantly, within the illustrated deformation space, the high- $K$ states all have significantly deeper and more rigid wells than the $0^{+}$states. However, it must be acknowledged that the calculation of the full barrier to fission out of the second well requires a larger deformation space, and it is sensitive to the details of the shape parameterization and to the procedures through which the fission path is obtained by minimizing the energy with respect to the shape parameters [22]. Realistic calculations of the high- $K$, configuration-constrained barriers need more detailed tests of these aspects. Nevertheless, the present calculations provide an important step, showing for the first time increased barrier heights to fission from second-well high- $K$ isomers. The inclusion of the $\beta_{3}$ degree of freedom is an essential aspect.

Fission is a complex dynamical process, and the fission lifetime is determined not only by the barrier but also by the dynamics. The description of the fission lifetime usually involves so-called inertial parameters [23]. In this respect, a broken-pair excitation results in a reduced paring gap, which leads to increased inertial parameters and hence to decreased fission probability [23]. This is also consistent with the experimental observations of relatively short-lived $0^{+}$fission isomers, compared with longer-lived high- $K$ fission isomers. (Indeed, the high- $K$ fission isomers in ${ }^{236,238} \mathrm{Pu}[6,24,25]$ and ${ }^{242,244} \mathrm{Cm}[26,25]$ were observed prior to the corresponding $0^{+}$fission isomers.) Building on the present implementation of the configuration constraint in the second well, further work is needed to determine the relative contributions of the increased barrier height and the pair-breaking effect to the extended half-lives. The en-

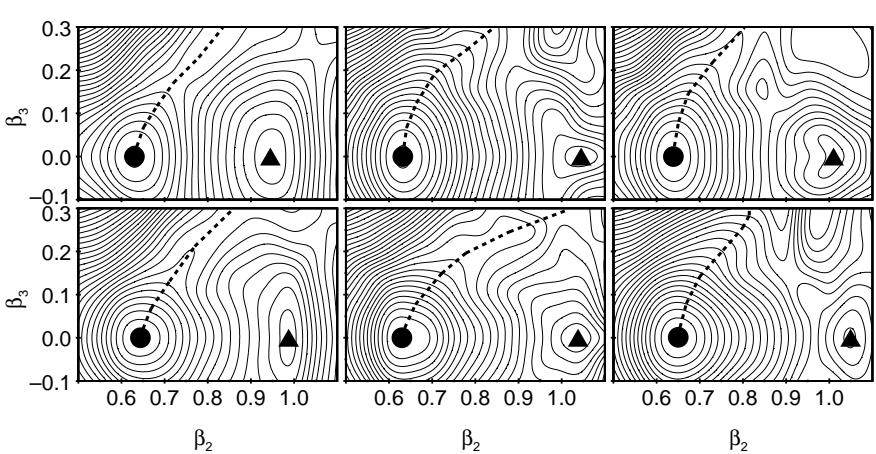

Fig. 2. Calculated $\beta_{3}$ vs. $\beta_{2}$ second-well PESs for the $0^{+}$fission isomer (top left), $K^{\pi}=8^{+}\{\nu 11 / 2[615] \otimes \nu 5 / 2[862]\}$ (top middle), and $K^{\pi}=8^{-}\{\pi 9 / 2[514] \otimes \pi 7 / 2[633]\}$ (top right) states in ${ }^{236} \mathrm{Pu}$, as well as the $0^{+}$fission isomer (bottom left), $K^{\pi}=7^{-}\{\nu 5 / 2[862] \otimes \nu 9 / 2[734]\}$ (bottom middle), and $K^{\pi}=10^{-}\{\nu 11 / 2[615] \otimes \nu 9 / 2[734]\}$ (bottom right) states in ${ }^{242} \mathrm{Cm}$. The filled circle (triangle) corresponds to the minimum (maximum) in the illustrated deformation space. The dashed line indicates the static fission path. The energy interval between neighbouring contours is $500 \mathrm{keV}$.

hanced stability of the high- $K$ fission isomers remains a remarkable situation which also merits more detailed experimental investigation.

In summary, using configuration-constrained PES calculations, including reflection-asymmetric shapes $\left(\beta_{3}\right.$ and $\left.\beta_{5}\right)$, we have investigated high- $K$ isomers in the second well for actinide nuclei, named high- $K$ fission isomers. Detailed insight into the properties of the states has been obtained, including configurations, deformations and excitation energies. For every observed high- $K$ fission isomer (all with unmeasured spin and parity) it is found that there are several possibilities for its configuration, with calculated energies close to the experimental value. The calculated PESs for the high- $K$ fission isomers show that the brokenpair structure can lead to an increased barrier against fission, compared with the corresponding $0^{+}$fission isomer. Additional experimental observations are needed to gain further understanding of the structures of isomers in the second well, and a wider configuration-constrained deformation space is needed before quantitative fission rates can be calculated.

This work has been supported by the Chinese Major State Basic Research Development Program under Grant 2007CB815000; the National Natural Science Foundation of China under Grants 10975006, 10735010 and 10875077; the Swedish Science Research Council (VR); and STFC and AWE plc (UK).

\section{References}

1. A. Bohr, B.R. Mottelson, Nuclear Structure, Vol. II, Benjamin, New York, 1975.

2. P.M. Walker, G.D. Dracoulis, Nature 399 (1999) 35.

3. S. Bjørnholm, J.E. Lynn, Rev. Mod. Phys. 52 (1980) 725.

4. B. Singh, R. Zywina, R.B. Firestone, Nucl. Data Sheets 97 (2002) 241; Evaluated Nuclear Structure Data File http:/www.nndc.bnl.gov/ensdf/. 
5. F.R. Xu, E.G. Zhao, R. Wyss, P.M. Walker, Phys. Rev. Lett. 92 (2004) 252501.

6. P. Limkilde, G. Sletten, Nucl. Phys. A 199 (1973) 504.

7. J.P. Delaroche, M. Girod, H. Goutte, J. Libert, Nucl. Phys. A 771 (2006) 103.

8. F.R. Xu, P.M. Walker, J.A. Sheikh, R. Wyss, Phys. Lett. B 435 (1998) 257.

9. H.L. Liu, F.R. Xu, S.W. Xu, R. Wyss, P.M. Walker, Phys. Rev. C 76 (2007) 034313.

10. S. Ćwiok, P. Rozmej, A. Sobiczewski, Z. Patyk, Nucl. Phys. A 491 (1989) 281.

11. I. Muntian, A. Sobiczewski, Phys. Lett. B 586 (2004) 254.

12. H.L. Liu, F.R. Xu, P.M. Walker, C.A. Bertulani, Phys. Rev. C 83 (2011) 011303(R).

13. P. Möller, J.R. Nix, Nucl. Phys. A 229 (1974) 269.

14. W. Nazarewicz, J. Dudek, R. Bengtsson, T. Bengtsson, I. Ragnarsson, Nucl. Phys. A 435 (1985) 397.

15. H.C. Pradhan, Y. Nogami, J. Law, Nucl. Phys. A 201 (1973) 357.

16. P. Möller, J.R. Nix, Nucl. Phys. A 536 (1992) 20.

17. W.D. Myers, W.J. Swiatecki, Nucl. Phys. 81 (1966) 1.

18. V.M. Strutinsky, Nucl. Phys. A 95 (1967) 420.

19. S. Raman, et al., At. Data Nucl. Data Tables 78 (2001) 1.

20. J. Schirmer, et al., Phys. Rev. Lett. 63 (1989) 2196.

21. J. Kantele, et al., Phys. Rev. Lett. 51 (1983) 91.

22. P. Möller, A.J. Sierk, T. Ichikawa, A. Iwamoto, R. Bengtsson, H. Uhrenholt, S. Åberg, Phys. Rev. C 79 (2009) 064304.

23. M. Brack, et al., Rev. Mod. Phys. 44 (1972) 320.

24. S.C. Burnett, H.C. Britt, B.H. Erkkila, W.E. Stein, Phys. Lett. B 31 (1970) 523.

25. H.C. Britt, S.C. Burnett, B.H. Erkkila, J.E. Lynn, W.E. Stein, Phys. Rev. C 4 (1971) 1444.

26. G. Sletten, V. Metag, E. Liukkonen, Phys. Lett. B 60 (1976) 153. 